\title{
Editorial
}

\section{Applications of Stochastic Processes in Biology and Medicine}

\author{
Charles J. Mode, ${ }^{1}$ Rick Durrett, ${ }^{2}$ Fima Klebaner, ${ }^{3}$ and Peter Olofsson ${ }^{4}$ \\ ${ }^{1}$ Department of Mathematics, Drexel University, Philadelphia, PA 19104, USA \\ ${ }^{2}$ Department of Mathematics, Duke University, Durham, NC, USA \\ ${ }^{3}$ School of Mathematical Sciences, Monash University, Melbourne, VIC, Australia \\ ${ }^{4}$ Department of Mathematics, Trinity University, San Antonio, TX, USA \\ Correspondence should be addressed to Charles J. Mode; cjmode@comcast.net
}

Received 27 February 2013; Accepted 27 February 2013

Copyright (C) 2013 Charles J. Mode et al. This is an open access article distributed under the Creative Commons Attribution License, which permits unrestricted use, distribution, and reproduction in any medium, provided the original work is properly cited.

Even though the title of this special edition suggests that a wide class of stochastic processes may be applicable in biology and medicine, all the six papers that were accepted for publication in this edition were based, either directly or indirectly, on a class of stochastic processes called branching processes. Within all the literature on probability, statistics and stochastic processes, papers, and books on branching processes occupy a relatively small but significant niche, even though their history spans and period of more than one hundred years. In particular, the papers that constitute this edition may be classified into three types; namely, models of epidemics of infectious diseases, genetics and evolution.

In a paper by $\mathrm{N}$. Champagnat et al. on birth and death process with neutral mutation, classes of branching processes are used to study mutations characterized in terms of splitting trees. It is interesting to note that mutations were not described in terms of nucleotide substitutions that are widely used in the classification of human haplotypes based on mitochondrial DNA. Like the analysis used in papers and books on branching processes, limit theorems played a basic role in the paper and the time scale of evolution was evolutionary time rather than generations.

In a paper by A. Denes et al. Galton-Watson multitype branching processes in discrete time with immigration were used to model risks of infectious disease by imported cases with applications to the European Football Championships of 2012. Among the many tools used by these authors in their analysis of the model was probability generating functions.

In the paper of S. C. Penisson and Jacob, the decay phase of the model for of an infectious disease caused by some infectious agent was approximated by a branching process.
Limit theorems again played a basic role in the analysis of the model. There is also an interesting application of the model to BSE, mad cow disease, epidemic in Great Britain.

A paper by V. Vatutin et al. is devoted to decomposable branching processes for the two-type case in, among other things, random environments. In particular, it is assumed that the population has two types. One type lives on one island with variable environments, and the other type lives on another island with a constant environment. Moreover, it is assumed that migration occurs only from a random environment to a constant environment. The main focus of the paper is to apply limit theorems to study probabilities of extinction in the critical and subcritical cases.

In a paper by C. J. Mode et al. the focus of attention is to formulate and partially analyze a model of the emergence of mutations and their subsequent evolution in an agestructured self-regulating stochastic process with two sexes. From the genetic point of view, only one autosomal locus with two alleles is considered. The working paradigm of the paper differs from that of other papers in the edition in that it is based on computer intensive methods, and, in particular, Monte Carlo simulation methods. The process is also self-regulating in the sense that the subpopulation of each genotype will attain a population size determined by the carrying capacity of the environment for that genotype. Another feature of this model is that, in contrast to most models of classical branching processes, a set of nonlinear deterministic equations is embedded in the stochastic process and the analysis of this deterministic system is part of the analysis of the model as a whole in computer simulation experiments. Interestingly, at some points in the parameter 
space of the process, the embedded deterministic model will exhibit chaotic behavior, but examples with chaos were not considered in the paper.

Lastly in a paper by $\mathrm{X}$. Wu and M. Kimmel, neutral evolution is modeled as an infinite allele Markov branching processes. The main focus of attention in this paper is the proportion of alleles having a given number of copies at some point in time, which is referred to as the frequency spectrum by the authors. A formula for the variance of the frequency spectrum is also derived by the authors that is useful in interval estimation and tests of hypotheses for parameter values. It is also shown that the mean of frequency spectrum has an explicit form expressed in terms of a hyper-geometric function. A formula for the asymptotic rate of convergence is also derived. A technique used in the derivation of the formulas involves probability generating functions. Simulations are also used to illustrate some of the results that were derived from the birth and death process.

Charles J. Mode Rick Durrett

Fima Klebaner Peter Olofsson 


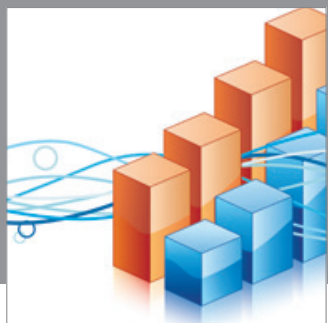

Advances in

Operations Research

mansans

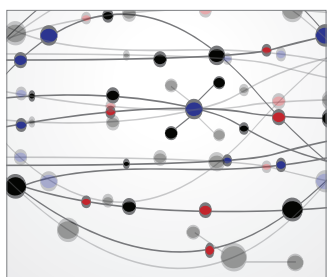

The Scientific World Journal
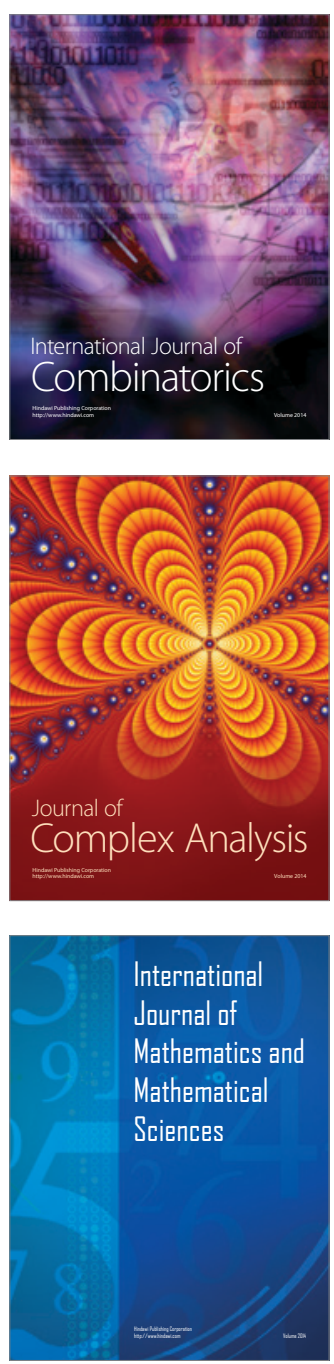
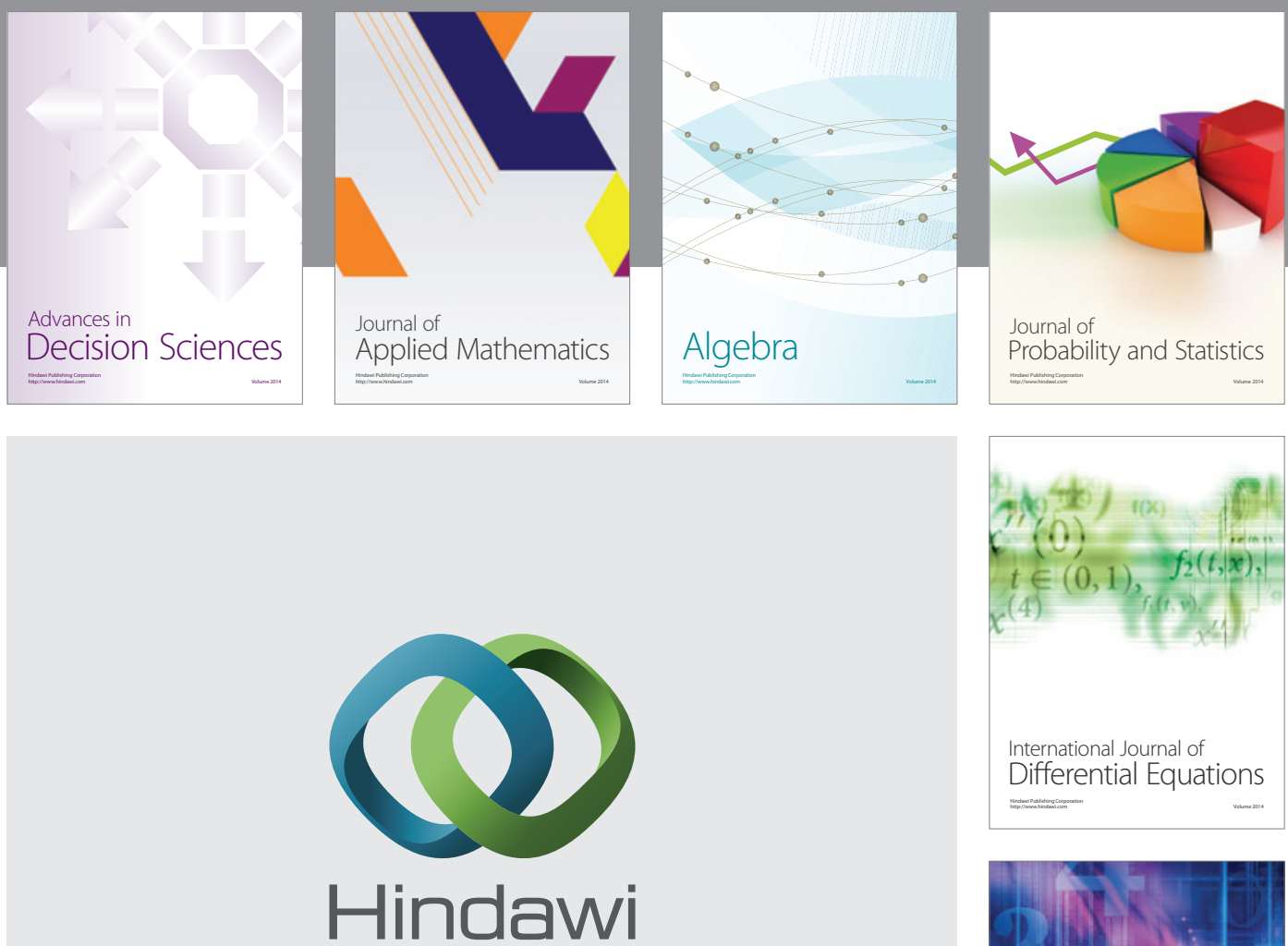

Submit your manuscripts at http://www.hindawi.com
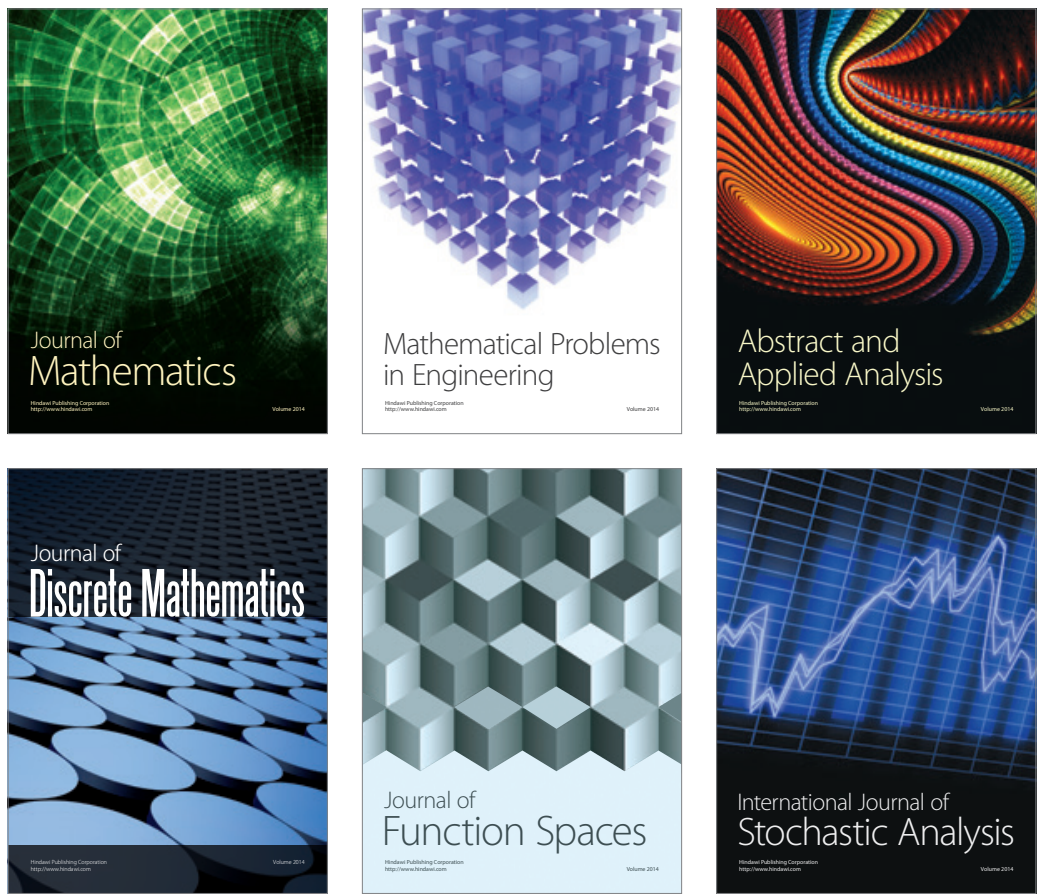

Journal of

Function Spaces

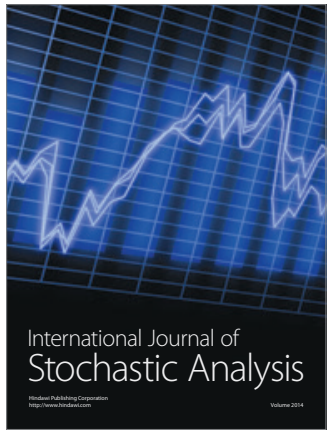

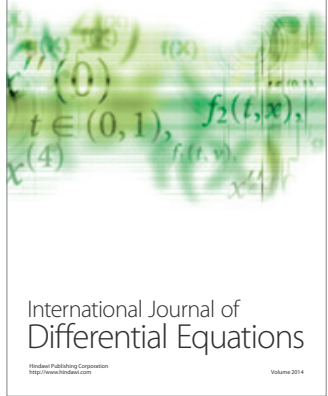
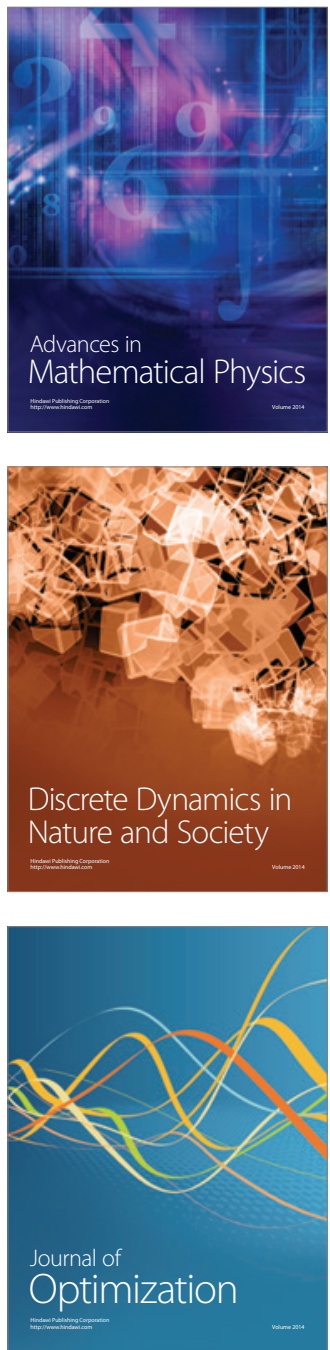